\title{
Three-dimensional Characterization of Selective Laser Melted Graphene Oxide- Reinforced Ti-48Al-2Cr-2Nb Alloy using FIB-SEM Tomography
}

Dian $\mathrm{Li}^{1}$, Wenrui Zhao ${ }^{1}$, Xing Zhang ${ }^{2}$, Yiliang Liao ${ }^{2}$ and Yufeng Zheng ${ }^{1}$

${ }^{1}$ University of Nevada Reno, United States, ${ }^{2}$ Iowa State University, United States

Additive manufacturing (AM) opens new avenues for manufacturing the near-net-shape TiAl components with complex geometries, which serve as promising structural material for aerospace application. Severe cracking remains as critical issue limiting the fabrication of TiAl alloys using selective laser melting (SLM). Our recent work indicated that decorating the AM powders with graphene oxide sheets can help solve the cracking issue associated with SLM of Ti-48Al-2Cr-2Nb (at.\%, Ti-4822) [1]. With the development of advanced characterization techniques, such as X-ray computed tomography, focused ion beam- scanning electron microscopy (FIB-SEM) tomography and atom probe tomography, the threedimensional (3D) features in the AM-built parts can investigated at various length scales. Due to the high space resolution, good site-specific precision and highly-efficient automated data collection, FIB-SEM tomography offers the unique opportunity to reveal the complex micro- to nano-scale structure and defects in the AM-built parts [2]. In the current work, we performed 3D characterization using FIB-SEM tomography to study the microstructure evolution and the defect formation during SLM of Ti-4822 and graphene oxide added $\mathrm{Ti}-4822$, and thus to investigate the influence of graphene oxide on the manufacturability of SLMedTiAl component.

In the first part of this work, the dual-phase microstructure ( $\alpha_{2}$ and $\gamma$ phases) in the as-printed Ti-4822 and the graphene oxide added Ti-4822 was investigated using 3D FIB-SEM tomography. FIB serial sectioning of 1000 slices with the slice thickness of $20 \mathrm{~nm}$ was performed in an area of $20 \mu \mathrm{m} \times 30 \mu \mathrm{m}$ at $30 \mathrm{kV}$ using FEI Scios 2 Dual-beam FIB/SEM, shown in Fig. 1(a). 3D reconstruction was conducted using MIPAR $^{\mathrm{TM}}$ image analysis software to achieve feature-by-feature quantification [3]. As shown in Fig. 1 (b), elongated honeycomb-like $\alpha_{2}$ grains formed along the printing direction was observed in the asprinted Ti-4822 together with the interconnected $\gamma$ phase networks. On the contrary, with the addition of graphene oxide, $\alpha_{2}$ cells become smaller while the $\gamma$ networks appear to be more tightly connected with thinner walls. Quantitative analysis reveals that with the addition of graphene oxide, the average volume of $\alpha_{2}$ cell reduces from $21.7 \mu^{3}$ to $11.8 \mu^{3}$, and $\gamma$ network thickness from $0.9 \mu \mathrm{m}$ to $0.5 \mu \mathrm{m}$. Thus, the 3D characterization using FIB-SEM tomography indicates a clear grain refinement, which is believed due to graphene oxide acting as barriers for the nucleation and growth of $\alpha_{2}$ phase during solidification.

The second part of this study focuses on 3D characterization of defects formed in the as-printed Ti-4822 and the graphene oxide added Ti-4822. Fig. 2(a) shows the defects formed in the as-printed Ti-4822, including multiple micro-scale cracks and sub-micro pores in different morphologies. In contrast, no cracks but only nano-scale pores with dramatically reduced size and number density are characterized in the graphene oxide added Ti-4822, shown in Fig. 2(b). Thus, our 3D characterization results have provided experimental evidence indicating that the graphene oxide can improve the manufacturability of Ti-4822.

D. Li, W. Zhao and Y. Zheng appreciate the financial support by the faculty startup funding from University of Nevada, Reno. 
(a)

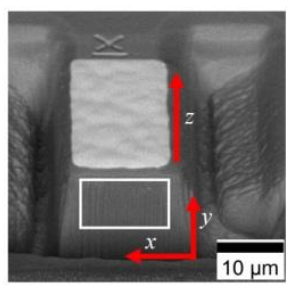

(b)

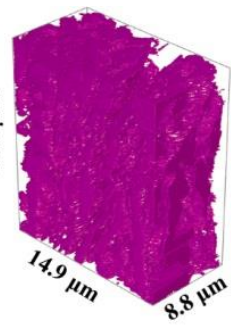

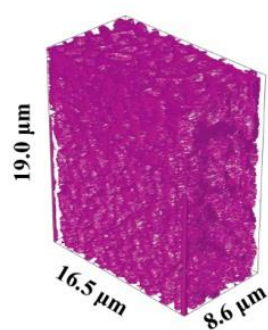

Figure 1. 3D characterization of microstructure in the SLMed Ti-4822 and the graphene oxide added Ti4822: (a) FIB-SEM tomography analyzed volume; and (b) 3D reconstruction of $\gamma$ phase in Ti-4822 and in the graphene oxide added Ti-4822.
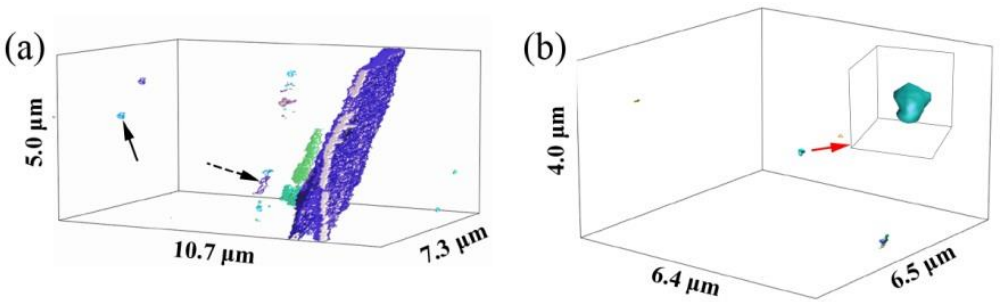

Figure 2. 3D characterization of defects in the Ti-4822 and the graphene oxide added Ti-4822: (a) the micro-scale crack and nano-scale pores from Ti-4822; and (b) nano-scale pores in the graphene oxide added Ti-4822.

References

[1] X. Zhang et al., Journal of Materials Research, 35 (2020) 1998-2005.

[2] R.E.A. Williams et al., Microscopy and Microanalysis, 20 (2014) 322-323

[3] J. Sosa et al., Integrating Materials and Manufacturing Innovation, 3 (2014) 123-140. 